\title{
Conformational studies on Arabidopsis sulfurtransferase AtStr1 with spectroscopic methods
}

\author{
Andrea Bartels', Fabio Forlani², Silvia Pagani² \\ and Jutta Papenbrock ${ }^{1, *}$ \\ ${ }^{1}$ Institut für Botanik, Universität Hannover, \\ Herrenhäuserstr. 2, D-30419 Hannover, Germany \\ ${ }^{2}$ Dipartimento di Scienze Molecolari Agroalimentari \\ (DISMA), Università di Milano, Via Celoria 2, I-20133 \\ Milano, Italy \\ *Corresponding author \\ e-mail: jutta.papenbrock@botanik.uni-hannover.de
}

\begin{abstract}
Sulfurtransferases/rhodaneses (Str) are enzymes widely distributed in archaea, prokaryota and eukaryota, and catalyze the transfer of sulfur from a donor molecule to a thiophilic acceptor substrate. In this reaction, Str cycles between the sulfur-free and the sulfur-substituted form. Two-domain Str consist of two globular domains of nearly identical size and conformation connected by a short linker sequence, which is elongated in plant two-domain Str proteins compared to Str in other organisms. The two-domain Arabidopsis thaliana Str1 protein (At1g 79230) was expressed in Escherichia coli as a mature protein, as a variant without the elongated linker sequence, and as AtStr1C332S and AtStr1C339V. The persulfuration state of the purified recombinant proteins was investigated in the presence and absence of sulfur donors by fluorescence spectroscopy. The secondary structure was analyzed by circular dichroism (CD) in the far-UV range, while overall changes in tertiary structure were determined by $C D$ in the near-UV range. Finally, protein stability was analyzed by tryptic digestion. The elongated linker sequence is essential for correct conformation and stability, and thereby affects the catalytic activity of AtStr1. Replacement of the catalytic cysteine residue C332 leads to higher rigidity of the molecule, whereas replacement of C339 does not lead to any conformational changes, providing evidence of the direct involvement of C339 in catalysis.
\end{abstract}

Keywords: Arabidopsis thaliana; circular dichroism; 3-mercaptopyruvate; mutagenesis; thiosulfate; tryptic digestion.

Sulfurtransferase (Str) enzymes catalyse the transfer of a sulfur atom from suitable sulfur donors to nucleophilic sulfur acceptors. The most studied and best-characterized Str is bovine rhodanese (thiosulfate:cyanide Str, EC 2.8.1.1), which catalyses the transfer of a sulfane sulfur atom from thiosulfate to cyanide in vitro, leading to the formation of sulfite and rhodanide (Westley, 1973). Solu- tion studies have identified three catalytic requirements: an active-site sulfhydryl group, two cationic residues, and a hydrophobic environment for substrate binding (Finazzi Agro et al., 1972; Miller-Martini et al., 1994a,b). However, in vivo neither donor substrates nor sulfur acceptors could be clearly identified in any of the organisms investigated.

Using different strategies to mine the databases, Strlike proteins containing typical rhodanese patterns or domains could be identified in Arabidopsis thaliana (Bauer and Papenbrock, 2002; Bartels and Papenbrock, unpublished results). Proteins containing rhodanese domains are structural modules found as single-domain proteins, as tandemly repeated modules in which only the C-terminal domain bears the properly structured active site, or as members of multi domain proteins. Two of the Arabidopsis Str forms, AtStr1 and AtStr2, belong to the two-domain Strs, consisting of two rhodanese domains ( $\mathrm{N}$ - and $\mathrm{C}$-terminal) of nearly identical size connected by a linker sequence. This overall structure is similar to the structure of bovine rhodanese; however, in plant two-domain Strs the connecting linker sequence is elongated in comparison to the linker of two-domain sequences from other eukaryotic organisms (Papenbrock and Schmidt, 2000b). The role of the elongated linker could not be clarified unambiguously so far. The AtStr1 sequence contains five cysteine residues, of which the catalytic cysteine residue $\mathrm{C} 332$ is the target of persulfide formation. In plant two-domain Strs, a second cysteine (C339) is located close to the catalytic cysteine; its function in catalysis and conformational stability have not been clarified yet.

Despite the presence of Str/rhodanese activities in all three domains of life, the physiological roles of the members of this multiprotein family have not been established unambiguously. Proposed roles include cyanide detoxification (Vennesland et al., 1982), sulfur metabolism (Donadio et al., 1990), and mobilization of sulfur for ironsulfur cluster biosynthesis or repair (Bonomi et al., 1977; Pagani et al., 1984). In plants, the mobilization of sulfur for transport processes in older leaves was also proposed (Papenbrock and Schmidt, 2000b).

Obligatory intermediates in Str/rhodanese catalysis, in the presence of the donor thiosulfate, are $E$ (sulfur-free enzyme) and ES (sulfur-substituted enzyme) forms. In the reaction, rhodanese cycles between the $\mathrm{E}$ and $\mathrm{ES}$ forms. The intrinsic fluorescence of rhodanese resulting from tryptophan residues is quenched when a stable persulfide is formed in ES due to energy transfer between the persulfide and the initially excited tryptophan residues, which can be followed by fluorescence spectroscopy.

Circular dichroism (CD) relies on the differential absorption of left and right circularly polarized radiation by chromophores that either possess intrinsic chirality 
Figure 1 Persulfuration states of AtStr1 and AtStr1wlink in the absence and presence of sulfur donors.

Fluorescence spectra (excitation at $280 \mathrm{~nm}$ ) of $3 \mu \mathrm{M}$ purified AtStr1 (A) and AtStr1wlink (B) in $20 \mathrm{~mm}$ Tris/ $\mathrm{HCl}, \mathrm{pH}$ 8.0, before (solid line) and after addition of a 10-fold molar excess of cyanide (KCN) (dashed line). Fluorescence spectra of $3 \mu \mathrm{M}$ AtStr1 $(\mathrm{C}, \mathrm{D})$ and AtStr1wlink (E,F) in $20 \mathrm{~mm}$ Tris/ $\mathrm{HCl}, \mathrm{pH}$ 8.0, before (solid line) and after (dotted line) addition of 10 -fold molar excess of 3-mercaptopyruvate $(\mathrm{C}, \mathrm{E})$ or thiosulfate $(\mathrm{D}, \mathrm{F})$. Both proteins were sulfane sulfur-deprived by cyanide treatment and excess reagent was removed by gel filtration before the fluorescence analysis. A.U., arbitrary fluorescence units.

Methods: AtStr1 protein and AtStr1 protein excluding the plant-specific linker sequence elongation (AtStr1wlink) were expressed and purified as previously described (Burow et al., 2002). Protein concentrations were determined according to Bradford (1976) using bovine serum albumin as a protein standard. Fluorescence measurements were performed using a LS50 spectrofluorimeter (Perkin Elmer, Monza, Italy) equipped with a thermostatic cell holder at $20^{\circ} \mathrm{C}$. The excitation wavelength was $280 \mathrm{~nm}$ in all experiments and the slit width for excitation and emission was 5 and $3 \mathrm{~nm}$, respectively. Proteins were used at a concentration of $3 \mu \mathrm{m}$ in $20 \mathrm{~mm}$ Tris/ $\mathrm{HCl}, \mathrm{pH}$ 8.0. Emission spectra were scanned from 300 to $400 \mathrm{~nm} 1 \mathrm{~min}$ after reagent addition and were corrected for dilutions due to reagent addition. The fluorescence intensities observed at $336 \mathrm{~nm}\left(F_{\text {obs }}\right)$ were calculated as $\Delta F(\%)$, defined as:

$\Delta F(\%)=-\frac{F_{\mathrm{obs}}-F_{0}}{F_{0}} \times 100$,

where $F_{0}$ is the original fluorescence intensity of the protein analyzed, as described by Colnaghi et al. (2001).

themselves or are placed in chiral environments. Proteins contain a number of chromophores that can give rise to CD signals. In the far-UV region (180-240 nm), the absorbing moiety is principally the peptide bond. Far-UV $C D$ analysis can be used to quantitatively assess the overall secondary structure content of the protein, because the different forms of regular secondary structure found in proteins generate different spectra (Kelly and Price, 2000). Near-UV CD detects aromatic amino acids constrained in rigid chiral environments, revealing their embedding in tertiary structure (Adler et al., 1973). Thus, $C D$ is an ideal technique to monitor conformational changes in proteins that can occur as a result of changes in experimental conditions or caused by mutations (Kelly and Price, 2000).

The aim of this study was to investigate the role of the unique plant linker sequence and the two most important of the five cysteine residues in AtStr1. Recombinant mutated proteins were expressed, purified and analyzed by different spectroscopic methods and compared to the mature wild-type protein.

First, the persulfuration status of recombinant AtStr1 and mutated forms was investigated. Formation of the covalent enzyme-sulfur intermediate ES can be detected by intrinsic fluorescence quenching of the enzyme due to energy transfer between the persulfide and the initially excited tryptophan residues (Horowitz and Criscimagna, 1983; Cannella et al., 1986). The fluorescence spectrum of purified AtStr1 changed following the addition of cyanide (Figure 1A). This nucleophilic acceptor could remove the sulfane sulfur from the persulfide bond to the cata-
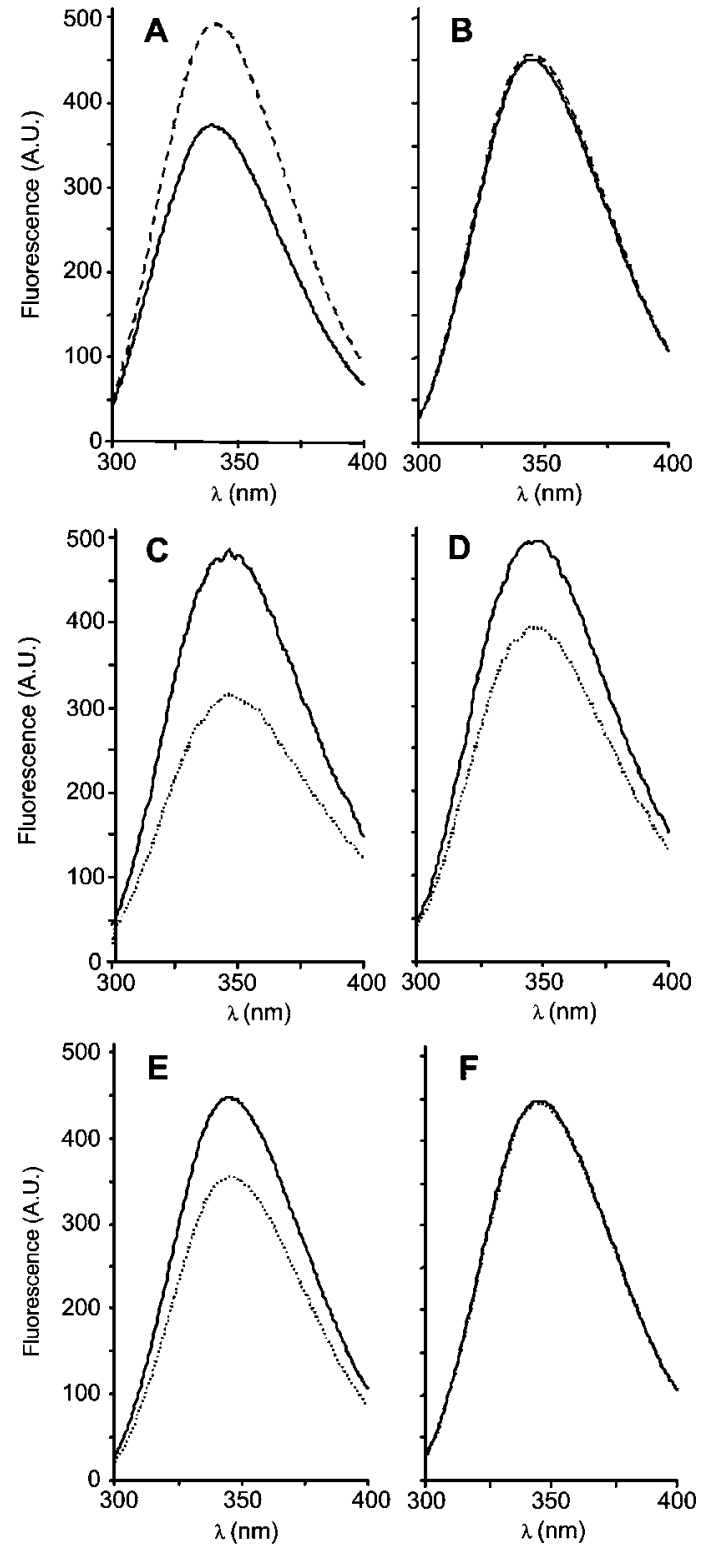

lytic cysteine. The fluorescence quenching observed indicates that approximately $80 \%$ of the recombinant AtStr1 full-length protein was isolated in a stable persulfurated form.

The AtStr1 protein showed Str activity in vitro using 3mercaptopyruvate (3-MP) and thiosulfate as sulfur donor substrates. The activity with thiosulfate was approximately six-fold lower; however, the $K_{\mathrm{m}}$ value for thiosulfate was lower than that for 3-MP (Papenbrock and Schmidt, 2000a; Burow et al., 2002). There is an obvious contradiction in the behavior of 3-MP and thiosulfate in the affinity to and binding by Str compared to their utilization as sulfur donor substrates. Therefore, the ability of 3-MP and thiosulfate to generate the sulfur-substituted ES form of AtStr 1 was monitored by determining the change in intrinsic fluorescence as a function of a 10-fold molar excess of the donor substrates (Figure 1C,D). Both substrates were able to generate the sulfur-substituted form of AtStr1.

The cysteine residue C332 in AtStr1 is homologous to bovine rhodanese $\mathrm{C} 247$, the catalytic cysteine that is the 
target of persulfide formation (Ploegman et al., 1979; Horowitz and Criscimagna, 1983). Close to this cysteine, a second cysteine residue C254 (C339 in plant Str) is located in two-domain eukaryotic Str. To investigate the impact of both cysteine residues on the persulfuration status, C332 was replaced by serine, as previously carried out for rat 3-MP Str (Nagahara and Nishino, 1996). In experiments performed by Miller-Martini et al. (1994b), cysteine residue C254 in bovine rhodanese was replaced by serine, resulting in enhanced sulfhydryl reactivity of the $E$ form. To exclude any specific influence of serine, we decided to replace C339 in AtStr1 by valine. Formation of the persulfurated intermediate is mandatory for sulfur transfer catalysis, and, as expected, the lack of thiosulfate and 3-MP Str activities in AtStr1C332S (Burow et al., 2002) paralleled the complete absence of persulfuration by thiosulfate and 3-MP. Enzyme activities of AtStr1C339V were reduced to approximately $80 \%$ with $3-\mathrm{MP}$ and to approximately $25 \%$ with thiosulfate. Different protein preparations of AtStr1C339V showed different results: either the protein was persulfurated directly after purification, as indicated by increased fluorescence after cyanide addition, or it could be persulfurated by thiosulfate or 3-MP, or it could not be persulfurated at all. Therefore, it is difficult to draw conclusions about the role of C339 during catalysis. Choice of the amino acid to replace cysteine might have an impact, because valine is larger size than cysteine and there could be steric alterations that influence the capability to bind 3-MP as substrate, which is larger than thiosulfate. On the other hand, enzyme activity measurements showed the protein was active with 3-MP used as substrate (Burow et al., 2002).

In all two-domain Strs identified so far, the N- and Cterminal domains are connected by a linker sequence. In plant two-domain Str sequences the, connecting linker sequence between both domains is approximately 20 aa longer than in various Str sequences from prokaryotic and other eukaryotic species. To obtain more information on the function of this linker sequence, a peptide of 23 aa was deleted from AtStr1 (AtStr1wlink). This deletion halved the specific enzyme activity with 3-MP in comparison to the specific activity of wild-type AtStr1. When thiosulfate was used, AtStr1wlink displayed only approximately one-third of the wild-type activity (Burow et al., 2002). It was previously shown that the activity of the mature AtStr1 protein including the elongated linker sequence was less affected by thermal denaturation and treatment with $4 \mathrm{M}$ urea than the recombinant protein without the linker (Burow et al., 2002). In this work we investigated AtStr1 protein without the linker elongation (AtStr1wlink) by spectroscopic methods in more detail. Fluorescence spectra showed that the ability of AtStr1wlink to form a stable persulfide group was almost completely lost (Figure 1B). In addition, fluorescence experiments indicated that formation of the persulfide group was achieved in the presence of 3-MP (Figure 1E), but not in the presence of thiosulfate (Figure 1F), in contrast to results obtained with AtStr1 (Figure 1C,D).

There were slight variations in the percentage persulfuration of the AtStr1 protein in diverse preparations, although the recombinant protein was always prepared and purified in the same way. The presence of different concentrations of thiols, such as cysteine, glutathione or DTT, in the dialysis buffer or directly added to the measurement cuvette did not influence the persulfuration state of AtStr1 (data not shown). The addition of cyanide in 10-fold molar excess or even in equimolar amounts showed that persulfide formation could be completely removed. After removal of cyanide by gel filtration, the protein could be persulfurated again in a concentrationdependent manner by both substrates chosen (3-MP and thiosulfate). Although the $K_{\mathrm{m}}$ for thiosulfate was lower than for 3-MP, a higher $V_{\max }$ was observed for 3-MP (Papenbrock and Schmidt, 2000a; Burow et al., 2002). The data from the present study could not resolve this contradiction. It can be concluded that the catalytically active cysteine in AtStr1 cycles in the reaction between the $E$ and the ES forms, as was hypothesized. It would be very interesting to compare active and inactive singledomain Str (AtStr14, AtStr15, AtStr16, and AtStr18) with respect to their persulfuration states and persulfide formation by different sulfur donors.

To obtain a deeper insight into the effects resulting from removal of the elongated linker peptide, we analyzed the conformational state of AtStr1wlink by CD spectroscopy in comparison to the wild-type and two mutated AtStr1 proteins (Figure 2). Any decrease in nearUV CD signal indicates a change in the position of the aromatic amino acids tryptophan $(\sim 290 \mathrm{~nm})$, tyrosine $(\sim 275-283 \mathrm{~nm})$, and phenylalanine $(\sim 255-270 \mathrm{~nm})$ on

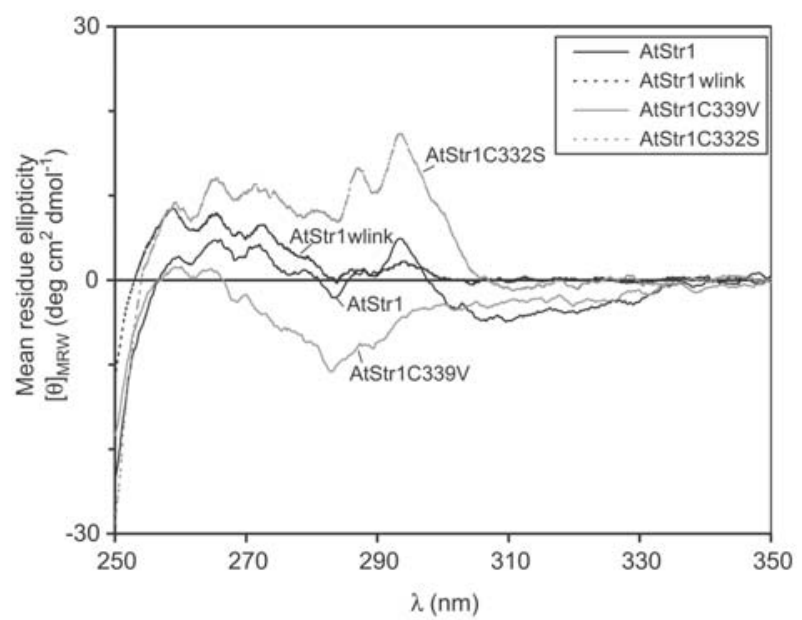

Figure 2 Near-UV CD spectra of AtStr1, AtStr1wlink, AtStr1C332S, and AtStr1C339V.

The spectra were recorded several times in $20 \mathrm{~mm}$ Tris $/ \mathrm{HCl}, \mathrm{pH}$ 8.0. AtStr1, $1.012 \mu \mathrm{g} \mu \mathrm{l}^{-1}$ (bold solid line); AtStr1wlink, $0.679 \mu \mathrm{g}$ $\mu \mathrm{l}^{-1}$ (bold dotted line); AtStr1C332S, $0.789 \mu \mathrm{g} \mu \mathrm{l}^{-1}$ (solid line); AtStr1C339V, $0.781 \mathrm{mg} \mathrm{\mu l}^{-1}$ (dotted line). Data deconvolution was performed using CDNN 2.1.

Methods: AtStr1, AtStr1wlink, AtStr1C332S, and AtStr1C339V were expressed and purified as previously described (Burow et al., 2002). Near-UV CD spectra were recorded at $25^{\circ} \mathrm{C}$ in a Jasco J-810 spectropolarimeter (Jasco, Great Dunmow, UK) at a scanning speed of $50 \mathrm{~nm} \mathrm{~min}{ }^{-1}$, a response of $0.5 \mathrm{~s}$, and bandwidth of $1 \mathrm{~nm}$. Data were collected at $0.2-\mathrm{nm}$ intervals in $0.1-\mathrm{cm}$ quartz cells from 250 to $350 \mathrm{~nm}$. Recorded spectra were corrected for the buffer baseline. Four accumulations were carried out for each spectrum. Protein amounts were determined spectrophotometrically at $280 \mathrm{~nm}$ using the extinction coefficient in the formula $1.55 \times A_{280}$, resulting in protein concentrations in $\mu \mathrm{g} \mu \mathrm{l}^{-1}$ (Layne, 1957). 
the surface of the protein and reflects a reduction in the order of tertiary structure. In addition, the formation of disulfide bridges leads to a weak signal at $260 \mathrm{~nm}$, which is often masked by signals of aromatic residues (Pouvreau et al., 2005).

The mature AtStr1 contains nine tryptophan residues mainly at the $\mathrm{N}$-terminus (six out of nine), eight tyrosine residues, 12 phenylalanine residues and five cysteine residues. AtStr1wlink contains one tyrosine residue fewer; the effects of the missing tyrosine residue are not predictable, but probably negligible. Interestingly, five of the six tryptophan residues in the N-terminus of AtStr1 are highly conserved in all eukaryotic sequences (Papenbrock and Schmidt, 2000b). Therefore, it can be assumed that their position in the tertiary structure is also highly conserved. This makes the near-UV CD technique particularly suitable for the analysis of conformational changes in the mutated proteins. AtStr1 displayed positive bands at 294, 288, 279, 272, 265, and $260 \mathrm{~nm}$ (Figure 2). AtStr1C332S displayed a sharper profile in the range of positive bands at 294 and $287 \mathrm{~nm}$, indicating a more rigid conformation of the tertiary structure in the regions of tryptophan residues. AtStr1C339V possesses a modified weakened tertiary structure relative to the other proteins. The signals were low, especially in the tyrosine-tryptophan region of the spectrum. The signal might be low because in the region $280-300 \mathrm{~nm}$ the signal cannot be well resolved, indicating the presence of multiple conformations due to the absence of rigidity, high flexibility or instability of the structure (Figure 2).

The spectrum of AtStr1wlink showed a decrease in the 294-nm signal relative to AtStr1. The loss of signal from tryptophan residues indicates that the AtStr1 linker is important for maintaining a compact conformational structure. CD measurements also showed that the AtStr1wlink signal was nearly zero in the range from 283 to $350 \mathrm{~nm}$, suggesting that lack of the linker peptide does not allow the native conformation. However, the overall structure of AtStr1wlink seems to be conserved, since there was little change in the phenylalanine spectrum. Because the proteins compared have the same number of aromatic amino acids, except for AtStr1wlink, the differences measured in the spectra can be attributed to the altered environment of the aromatic amino-acid residues.

For the AtStr1wlink protein, differences mainly in the shape of the CD spectrum could be observed. This indicates a more dynamic and flexible and consequently less stable tertiary structure of the protein. Removal of the plant-specific linker led to a reduction in enzyme activity to approximately $50 \%$ for $3-\mathrm{MP}$ and $30 \%$ for thiosulfate (Burow et al., 2002). Obviously, the linker sequence plays an important role not only in providing a hydrophobic environment for substrate binding and catalysis, as previously shown (Finazzi Agro et al., 1972; Miller-Martini et al., 1994a,b), but is also highly responsible for the generation of an ordered tertiary structure of the AtStr1 protein. Due to the removal of the linker elongation, the protein loses tight packing of the tertiary structure and thus adopts the structural features of the so-called molten globule state. It is noteworthy that although no tight tertiary structure was detected, the percentage second- ary structure of the protein often remains comparable to the wild-type protein (Buhot et al., 2004), as in the case of AtStr1wlink.

$C D$ in the far-UV range can give information about the percentage secondary structure of a protein. The spectra recorded between 190 and $260 \mathrm{~nm}$ revealed differences in the curve shape of AtStr1wlink in comparison to the three other proteins (Figure 3). Spectra of these three proteins were characterized by a positive signal below $200 \mathrm{~nm}$, a minimum at $208 \mathrm{~nm}$, and a shoulder at $225 \mathrm{~nm}$, indicating that they adopted an ordered structure according to Buhot et al. (2004), whereas the spectrum of AtStr1wlink did not show a minimum at $208 \mathrm{~nm}$ nor a shoulder at $225 \mathrm{~nm}$.

The percentage secondary structure was calculated according to Bohm et al. (1992) using the CDNN 2.1 program. This program is based on neural network analysis and compares the CD spectrum of the unknown protein with reference spectra of proteins with known secondary structure. The helical contents calculated are very similar for AtStr1, AtStr1C332S and AtStr1C339V (27\%), in agreement with the helical content calculated from the three-dimensional structure of Azotobacter vinelandii RhdA (25\%) (Bordo et al., 2000). The helical content of AtStr1wlink was significantly lower (20\%), indicating shifting and disorganization of secondary structural elements in this protein. The percentage $\beta$-sheet was very similar in all four recombinant proteins analyzed (18\%).

A computer program for prediction of protein secondary structure (GORIV, Garnier et al., 1996) revealed that the 16 amino acids in the core of the elongated linker in plant two-domain Str forms have a high probability of an $\alpha$-helix secondary structure. This prediction was confirmed experimentally: the C-terminal domain of AtStr1 with and without the extended linker sequence was

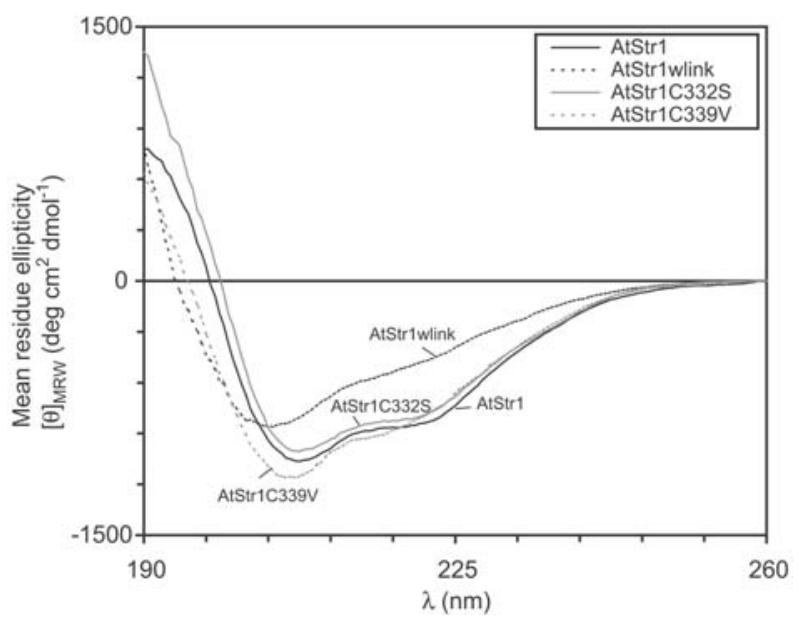

Figure 3 Far-UV CD spectra of AtStr1, AtStr1wlink, AtStr1C332S, and AtStr1C339V.

Spectra were recorded in $20 \mathrm{~mm}$ Tris/ $\mathrm{HCl}, \mathrm{pH}$ 8.0. The concentration and different numbers of amino acids were taken into consideration.

Methods: Far-UV CD spectra were recorded at $25^{\circ} \mathrm{C}$ at a scan rate of $5 \mathrm{~nm} \mathrm{~min}{ }^{-1}$, response of $0.25 \mathrm{~s}$, and bandwidth of $1 \mathrm{~nm}$, and were corrected for the buffer baseline. Data were collected at $0.1-\mathrm{nm}$ intervals from 190 to $260 \mathrm{~nm}$. Analyses were performed at a protein concentration of $3 \mu \mathrm{M}$. At least three accumulations were carried out for each spectrum. Protein concentrations were determined according to Bradford (1976). 
expressed and purified and CD results in the far UVrange demonstrated a higher helix content in the C-terminus including the linker in comparison to the C-terminus alone (data not shown). Extended BLAST searches could not identify any sequence similarities of the plant linker sequence with other proteins.

The linker in AtStr1 seems to play a different role than the shorter linker sequences usually found in two-domain eukaryotic Strs. The alignment of several two-domain eukaryotic Strs demonstrated the exceptional role of elongated plant linkers (Papenbrock and Schmidt, 2000b). Two-domain Str proteins from plants are localized in the cytoplasm (AtStr2) and in mitochondria (AtStr1) (Bauer et al., 2004). The exact subcellular localization of AtStr1 within mitochondria is not yet known. To the best of our knowledge, there are no other mitochondrial proteins containing elongated linker sequences connecting two domains. Therefore, comparisons with other proteins are not possible. The $\mathrm{pH}$ in the mitochondria is approximately 7.5 , which is much higher than the predicted $\mathrm{pl}$ of AtStr1 ( $\mathrm{pl}$ 5.1). In our studies, a value close to the mitochondrial $\mathrm{pH}$ was chosen to imitate the in vivo conditions. Deletion of the linker sequence and point mutations of the cysteine residues had a negligible influence on the $\mathrm{p} /$ values of the four recombinant proteins. A decrease to $\mathrm{pH} 5.0$ changed the spectroscopic results only slightly.

As CD spectroscopy revealed altered conformations of the recombinant AtStr1 mutants investigated, the consequences on susceptibility to proteolytic digestion was analyzed. In previous experiments, sensitivity to thermal denaturation and incubation with urea were determined only by activity measurements (Burow et al., 2002). Application of this third method might reveal whether wildtype and mutant proteins differ in their accessibility to proteolysis due to conformational changes caused by the mutations. Trypsin specifically digests proteins at the carboxy terminus of the basic amino acids lysine and arginine. AtStr 1 contains 17 lysine and 14 arginine residues, and therefore a very large number of fragments after even partial tryptic proteolysis is expected. AtStr1wlink contains only 15 lysine and also 14 arginine residues, and therefore almost the same number of peptide fragments can be expected.

Samples of $1 \mu \mathrm{g}$ of each protein preparation (AtStr1, AtStr1wlink, AtStr1C339V and AtStr1C332S) were digested by trypsin. At different time points $(0,15,30$, 45 , and $60 \mathrm{~min}$ ), samples were taken, as well as a control without trypsin at $60 \mathrm{~min}$, and analyzed by SDS-PAGE and Western blotting (Figure 4). For the concentration used, the trypsin band was not visible in Coomassiestained gels. To exclude any cross-reactivity of the RGS antibody with trypsin, a $2-\mu \mathrm{g}$ trypsin aliquot, approximately 40-fold more concentrated than that used for digestion, was loaded onto an SDS gel and analyzed by Western blotting. The 24-kDa trypsin band was not recognized by the RGS antibody (data not shown).

The mature AtStr1 protein has a predicted molecular mass of $35.5 \mathrm{kDa}$, including the $\mathrm{His}_{6}$-tag. Within the first $15 \mathrm{~min}$, approximately $50 \%$ of the protein was digested to smaller fragments of $\sim 32,29$ and $27 \mathrm{kDa}$ (Figure $4 \mathrm{~A}, \mathrm{~B})$. As observed in the Coomassie Blue-stained gel,
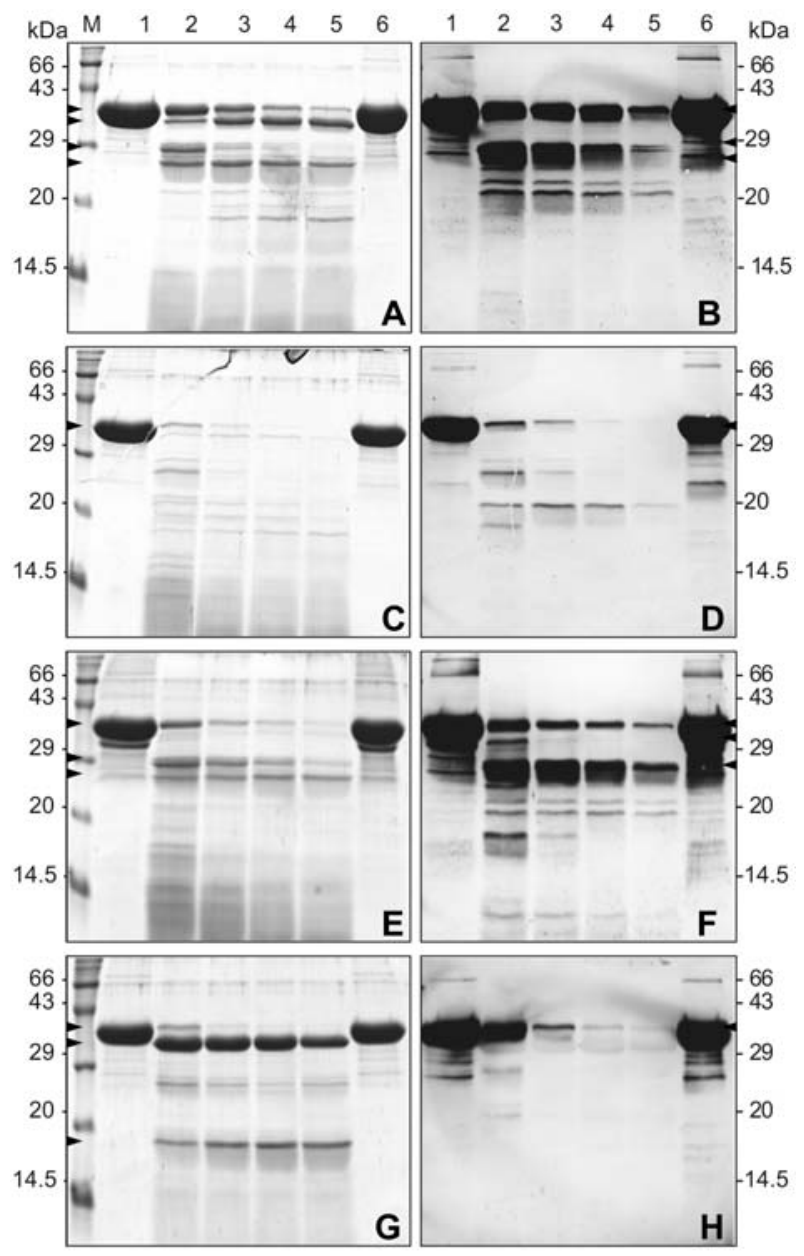

Figure 4 Partial proteolysis by trypsin digestion.

Coomassie Brilliant Blue-stained gels (A,C,E, G) and Western blot analysis $(B, D, F, H)$. From left to right: lane $M$, protein standard; lane 1, before digestion; lane 2, 15-min; lane 3, 30-min; lane 4, 45-min; and lane 5, 60-min digestion; lane 6, 60 min without trypsin. (A,B) AtStr1; (C,D) AtStr1wlink; (E,F) AtStr1C339V; (G,H) AtStr1C332S. Representative results from three sets of experiments are shown.

Methods: Affinity-purified proteins $\left(1 \mu \mathrm{g} \mu \mathrm{l}^{-1}\right)$ in $20 \mathrm{~mm}$ Tris/ $\mathrm{HCl}$, pH 8.0, were proteolyzed with $0.5 \%$ trypsin (Serva, Heidelberg, Germany) at $23^{\circ} \mathrm{C}$. The assay also contained $2 \mathrm{~mm}$ $\mathrm{CaCl}_{2}$ to avoid autolysis of trypsin. The proteolysis was monitored by SDS-PAGE over a time scale of $60 \mathrm{~min}$. The digestion was stopped at the time points indicated by adding $9 \mu$ l of $4 \times$ SDS-PAGE sample buffer $(2.4 \mathrm{ml}$ of mercaptoethanol, $6 \mathrm{ml}$ of $40 \%$ SDS, $240 \mu \mathrm{l}$ of bromophenol blue, $8 \mathrm{ml}$ of glycerol, $3.6 \mathrm{ml}$ of $1 \mathrm{M} \mathrm{Tris} / \mathrm{HCl}, \mathrm{pH} 6.8$, in a total volume of $20 \mathrm{ml}$ ) to $15-\mu \mathrm{l}$ aliquots of the assay and boiling the samples for $10 \mathrm{~min}$. SDSPAGE was performed according to Laemmli (1970) using 5\% acrylamide gels. Gels were stained with Coomassie Brilliant Blue or used for Western blotting. Proteins were transferred to nitrocellulose membranes (Roth, Karlsruhe, Germany) by the semidry method and immobilized for immunodetection. Western blot analysis was performed according to Sambrook et al. (1989) using an RGS antibody from mouse recognizing the N-terminal $\mathrm{His}_{6}$-tag of the recombinant proteins (Qiagen, Hilden, Germany), anti-mouse antibody from rabbit (Sigma, Taufkirchen, Germany) and anti-rabbit antibody conjugated with alkaline phosphatase (Sigma). Colorimetric detection was carried out using nitroblue tetrazolium and 5-bromo-4-chloro-3-indolyl-phosphate.

the amount of the 32-kDa fragment continuously increased (Figure 4A). This fragment did not contain the $\mathrm{His}_{6}-$ tag, as observed by immunoblot analysis (Figure 4B). 
Western blot analysis revealed that the band at $29 \mathrm{kDa}$ is predominant at the beginning of the proteolysis, and then decreased rapidly within 60 min of incubation to almost undetectable amounts. Part of the mature protein remained undigested, even after 60 min of trypsin treatment. A small percentage of all four protein preparations seemed to dimerize, as observed in Western blot analysis (Figure 4B,D,F,H). Immediately after trypsin addition, the homodimers disappeared.

AtStr1wlink has a predicted molecular mass of 33.3 $\mathrm{kDa}$. The difference in size in comparison to AtStr1 can be observed in the SDS-PAGE analysis (Figure 4C,D). The AtStr1wlink protein was proteolyzed by trypsin to a very large number of smaller protein fragments within 15 min of digestion. It seemed to be highly prone to degradation by a protease, in agreement with results obtained previously (Burow et al., 2002). As early as after $45 \mathrm{~min}$, none of the intact protein could be detected, even by very sensitive Western blot analysis (Figure 4D). Progress of the proteolysis of AtStr1C339V (Figure 4E,F) was comparable to the results obtained for mature AtStr1 (Figure 4A,B). The degradation seemed to progress slightly faster than for AtStr1. The AtStr1C332S protein was immediately digested to a band of approximately $32 \mathrm{kDa}$ (Figure 4G,H). This degradation product and a 17$\mathrm{kDa}$ fragment were relatively stable up to the end of the trypsin incubation. N-Terminal fragments containing the $\mathrm{His}_{6}$-tag were almost undetectable as early as after 45 min of trypsin treatment. In summary, the results indicate that AtStr1wlink can be easily digested by trypsin. This high susceptibility might explain the highly reduced enzyme activity (up to 50\%). By removal of the linker sequence, both domains are probably not able to adopt the correct positioning in relation to each other. Therefore, trypsin cleavage sites usually buried inside the protein are more accessible to the endoprotease than in the wild-type protein. AtStr1wlink gave the lowest signals in near-UV CD analysis. Both independent results indicate high flexibility of the tertiary structure, although the overall percentage of secondary structures is almost unchanged. As a consequence of site-directed mutagenesis in the linker sequence of bovine rhodanese, the protein was virtually indistinguishable from the wild-type protein in all properties tested, except for an increased susceptibility to perturbation (Luo et al., 1995). Elongation of the linker during evolution of the plant protein was obviously essential to provide full enzyme activity for plant-specific substrates.

In the proteolysis studies, an antibody directed against the $\mathrm{His}_{6}$-tag was used for Western blot analysis because this antibody recognized all four recombinant proteins with comparable sensitivity. The polyclonal antibody directed against the recombinant AtStr1 protein (Papenbrock and Schmidt, 2000a) showed a weaker reaction with AtStr1wlink than with the other three proteins (data not shown). Since all four recombinant proteins analyzed contained a $\mathrm{His}_{6}$-tag at the $\mathrm{N}$-terminus, only the $\mathrm{N}$-terminal part including the $\mathrm{His}_{6}$-tag can be detected by the RGS antibody. Therefore, conclusions can be drawn about different susceptibilities of either the $\mathrm{N}$ - or C-terminus using this antibody. In all four proteins analyzed, the N-terminus showed higher accessibility for trypsin digestion than the C-terminus. In addition, the two domains of $A$. vinelandii rhodanese show different resistance to endoproteinases, although in this case the Cterminus is more prone to digestion than the $\mathrm{N}$-terminal domain over time (Melino et al., 2004).

So far, no data concerning protein stability of AtStr1C332S have been observed. The methods used in previous studies based on determination of enzyme activity showed that replacement of the catalytically active cysteine C332 led to a complete loss of activity (Burow et al., 2002). Therefore, the method of partial tryptic digestion could give new insights into the role of C332 in the overall stability of AtStr1C332S. The first degradation product of AtStr1C332S is quite stable, and the overall stability and rigidity seems to be higher than for the wild-type protein (Figure 4A,G). This result is in agreement with the near-UV CD analysis, in which the strongest signals were found for AtStr1C332S, indicating high rigidity of the molecule. The increased rigidity associated with lack of the catalytic residue C332 could be due to the loss of distinct conformational states revealed in other Strs, e.g., RhdA from A. vinelandii (Bordo et al., 2000).

AtStr1C339V has a slightly modified tertiary structure relative to the other proteins. The signals are low especially, in the tyrosine-tryptophan region of the spectrum, probably because at $\mathbf{2 8 0}-300 \mathrm{~nm}$ the signal is not well resolved, indicating the presence of multiple conformations due to the absence of rigidity, high flexibility or instability of the structure (Figure 2). The tryptic digestion pattern of AtStr1C3339V shows high similarity to that of AtStr1, indicating similar accessibility of digestion sites and comparable conformation, in agreement with results obtained by spectroscopy. In a previous study, mutation of all non-essential cysteine residues in bovine rhodanese to serines changed the enzyme to a form (C3S) that was fully active, but less stable than the wild type. In 1,1'bis(4-anilino)naphthalene-5, $5^{\prime}$-disulfonic acid (bis-ANS) binding studies, it was shown that more hydrophobic regions in C3S were exposed on the surface of the protein than in the wild type, although both had similar secondary structures, suggesting a flexibility of its structure. bis-ANS bound to the C3S C-terminal domain, as shown by gel electrophoresis and proteolysis, and binding made the C-terminal domain more susceptible to trypsin cleavage (Kaur et al., 2004). Although rhodanese proteins display high sequence homology to each other, they differ significantly in their physico-biochemical behavior. Detailed experimental investigations are individually necessary for each protein.

For final conclusions on the impact of conformational changes on catalysis, the three-dimensional structures of the wild-type and mutated proteins need to be determined experimentally by X-ray analysis of protein crystals in the presence of substrates or analogs. So far, only the three-dimensional structure of the non-catalytic rhodanese homology domain of AtStr4 (At4g01050) has been resolved (Pantoja-Uceda et al., 2005).

In summary, the complete loss of enzyme activity in AtStr1C332S previously determined (Burow et al., 2002) can be explained by the missing catalytically active cysteine, but is also caused by conformational changes in the protein structure. AtStr1C339V protein shows almost 
no differences in the overall secondary and tertiary structure, and therefore the reduced enzyme activity (Burow et al., 2002) indicates a direct involvement of C339 in the catalytic mechanism. The reduced activity of AtStr1wlink is clearly based on higher flexibility and reduced stability of the protein molecule, indicating the importance of the elongated linker sequence for correct conformation of the protein.

\section{Acknowledgments}

We would like to thank Pamela von Trzebiatowski for her excellent technical assistance. This work was financially supported by Deutsche Forschungsgemeinschaft (PA 764/1-4).

\section{References}

Adler, A.J., Greenfield, N.J., and Fasman, G.D. (1973). Circular dichroism and optical rotatory dispersion of proteins and polypeptides. Methods Enzymol. 27, 675-735.

Bauer, M. and Papenbrock, J. (2002). Identification and characterization of single-domain thiosulfate sulfurtransferases from Arabidopsis thaliana. FEBS Lett. 532, 427-431.

Bauer, M., Dietrich, C., Nowak, K., Sierralta, W.D., and Papenbrock, J. (2004). Intracellular localization of sulfurtransferases from Arabidopsis thaliana. Plant Physiol. 135, 916-926.

Bohm, G., Muhr, R., and Jaenicke, R. (1992). Quantitative analysis of protein far UV circular dichroism spectra by neural networks. Protein Eng. 5, 191-195.

Bonomi, F., Pagani, S., Cerletti, P., and Canella, C. (1977). Rhodanese-mediated sulfur transfer to succinate dehydrogenase. Eur. J. Biochem. 72, 17-24.

Bordo, D., Deriu, D., Colnaghi, R., Carpen, A., Pagani, S., and Bolognesi, M. (2000). The crystal structure of a sulfurtransferase from Azotobacter vinelandii highlights the evolutionary relationship between the rhodanese and phosphatase enzyme families. J. Mol. Biol. 298, 691-704.

Bradford, M.M. (1976). A rapid and sensitive method for the quantification of microgram quantities of protein utilizing the principle of protein-dye binding. Anal. Biochem. 72, 248254.

Buhot, C., Chenal, A., Sanson, A., Pouvelle-Moratille, S., Gelb, M.H., Menez, A., Gillet, D., and Maillere, B. (2004). Alteration of the tertiary structure of the major bee venom allergen Apim1 by multiple mutations is concomitant with low IgE reactivity. Protein Sci. 13, 2970-2978.

Burow, M., Kessler, D., and Papenbrock, J. (2002). Enzymatic activity of the Arabidopsis sulfurtransferase resides in the Cterminal domain but is boosted by the $\mathrm{N}$-terminal domain and the linker peptide in the full length enzyme. Biol. Chem. 383, 1363-1372.

Cannella, C., Berni, R., Rosato, N., and Finazzi-Agro, A. (1986). Active site modifications quench intrinsic fluorescence of rhodanese by different mechanisms. Biochemistry 25, 73197323.

Colnaghi, R., Cassinelli, G., Drummond, M., Forlani, F., and Pagani, S. (2001). Properties of the Escherichia coli rhodanese-like protein SseA: contribution of the active-site residue Ser240 to sulfur donor recognition. FEBS Lett. 500, 153-156.

Donadio, S., Shafiee, A., and Hutchinson, R. (1990). Disruption of a rhodanese like gene results in cysteine auxotrophy in Saccharopolyspora erythraea. J. Bacteriol. 172, 350-360.

Finazzi Agro, A., Federici, G., Giovagnoli, C., Cannella, C., and Cavallini, D. (1972). Effect of sulfur binding on rhodanese fluorescence. Eur. J. Biochem. 28, 89-93.
Garnier, J., Gibrat, J.F., and Robson, B. (1996). GOR method for predicting protein secondary structure from amino acid sequence. Methods Enzymol. 266, 540-553.

Horowitz, P. and Criscimagna, N.L. (1983). The use of intrinsic protein fluorescence to quantitate enzyme-bound persulfide and to measure equilibria between intermediates in rhodanese catalysis. J. Biol. Chem. 258, 7894-7896.

Kaur, Y., Ybarra, J., and Horowitz, P.M. (2004). Active rhodanese lacking nonessential sulfhydryl groups has increased hydrophobic exposure not observed in wild-type enzyme. Protein J. 23, 255-261.

Kelly, S.M. and Price, N.C. (2000). The use of circular dichroism in the investigation of protein structure and function. Curr. Protein Pept. Sci. 1, 349-384.

Laemmli, U.K. (1970). Cleavage of structural proteins during the assembly of the head of bacteriophage T4. Nature 227, 680-685.

Layne, E. (1957). Spectrophotometric and turbidimetric methods for measuring proteins. Methods Enzymol. 3, 447-455.

Luo, G.X., Hua, S., and Horowitz, P.M. (1995). Mutation in the interdomain tether influences the stability and refolding of the enzyme rhodanese. Biochim. Biophys. Acta 1252, 165-171.

Melino, S., Cicero, D.O., Forlani, F., Pagani, S., and Paci, M. (2004). The N-terminal rhodanese domain from Azotobacter vinelandii has a stable and folded structure independently of the C-terminal domain. FEBS Lett. 577, 403-408.

Miller-Martini, D.M., Chirgwin, J.M., and Horowitz, P.M. (1994a). Mutations of noncatalytic sulfhydryl groups influence the stability, folding, and oxidative susceptibility of rhodanese. J. Biol. Chem. 269, 3423-3428.

Miller-Martini, D.M., Hua, S., and Horowitz, P.M. (1994b). Cysteine 254 can cooperate with active site cysteine 247 in reactivation of 5,5'-dithiobis(2-nitrobenzoic acid)-inactivated rhodanese as determined by site-directed mutagenesis. J. Biol. Chem. 269, 12414-12418.

Nagahara, N. and Nishino, T. (1996). Role of amino acid residues in the active site of rat liver mercaptopyruvate sulfurtransferase. cDNA cloning, overexpression, and site-directed mutagenesis. J. Biol. Chem. 271, 27395-27401.

Pagani, S., Bonomi, F., and Cerletti, P. (1984). Enzymic synthesis of the iron-sulfur cluster of spinach ferredoxin. Eur. J. Biochem. 142, 361-366.

Pantoja-Uceda, D., Lopez-Mendez, B., Koshiba, S., Inoue, M., Kigawa, T., Terada, T., Shirouzu, M., Tanaka, A., Seki, M., Shinozaki, K., et al. (2005). Solution structure of the rhodanese homology domain At4g01050(175-295) from Arabidopsis thaliana. Protein Sci. 14, 224-230.

Papenbrock, J. and Schmidt, A. (2000a). Characterization of a sulfurtransferase from Arabidopsis thaliana. Eur. J. Biochem. 267, 145-154.

Papenbrock, J. and Schmidt, A. (2000b). Characterization of two sulfurtransferase isozymes from Arabidopsis thaliana. Eur. J. Biochem. 267, 5571-5579.

Ploegman, J.H., Drent, G., Kalk, K.H., and Hol, W.G. (1979). The structure of bovine liver rhodanese. II. The active site in the sulfur-substituted and the sulfur-free enzyme. J. Mol. Biol. 127, 149-162.

Pouvreau, L., Kroff, T., Gruppen, H., van Koningsveld, G., van den Broek, L.A.M., and Voragen, A.G.J. (2005). Structure and stability of the potato cysteine protease inhibitor group (cv. Elkana). J. Agric. Food Chem. 53, 5739-5746.

Sambrook, J., Fritsch, E.F., and Maniatis, T. (1989). Molecular Cloning: A Laboratory Manual, $2^{\text {nd }}$ Edition (Cold Spring Harbor, NY, USA: Cold Spring Harbor Laboratory Press).

Vennesland, B., Castric, P.A., Conn, E.E., Solomonson, L.P., Volini, M., and Westley, J. (1982). Cyanide metabolism. Fed. Proc. 41, 2639-2648.

Westley, J. (1973). Rhodanese. Adv. Enzymol. 39, 327-368.

Received May 26, 2006; accepted August 28, 2006 